$\mid$ Pbilosophia Philosophia Scientiæ

Scientie Travaux d'histoire et de philosophie des sciences

$19-2$ | 2015

Circulations et échanges mathématiques

\title{
Le microcosme de la géométrie souterraine : échanges et transmissions en mathématiques pratiques
}

Thomas Morel

\section{(2) OpenEdition}

Journals

Édition électronique

URL : http://journals.openedition.org/philosophiascientiae/1089

DOI : 10.4000/philosophiascientiae.1089

ISSN : $1775-4283$

Éditeur

Éditions Kimé

Édition imprimée

Date de publication : 25 mai 2015

Pagination : $17-36$

ISSN : $1281-2463$

Référence électronique

Thomas Morel, «Le microcosme de la géométrie souterraine : échanges et transmissions en mathématiques pratiques », Philosophia Scientiæ [En ligne], 19-2 | 2015, mis en ligne le 19 juin 2015, consulté le 05 novembre 2020. URL : http://journals.openedition.org/philosophiascientiae/1089 ; DOI : https://doi.org/10.4000/philosophiascientiae.1089 


\title{
Le microcosme de la géométrie souterraine : échanges et transmissions en mathématiques pratiques
}

\author{
Thomas Morel \\ Technische Universität Berlin (Allemagne)
}

Résumé : La géométrie souterraine est une science mathématique pratique, qui se développe dans les exploitations minières et dont la diffusion est considérablement modifiée au cours du XVIII ${ }^{\mathrm{e}}$ siècle. Ce phénomène est lié à l'institutionnalisation graduelle de la discipline, de l'établissement d'un système de compagnonnage à la création d'académies des mines. Progressivement, les pratiques vont faire appel à de nouvelles méthodes et intégrer une solide formation en mathématiques théoriques. La circulation et l'enseignement des connaissances sont dans un premier temps basés sur un système de manuscrits qui sera étudié en détail. L'exemple du Markscheider August Beyer (1677-1753) illustre ce processus collectif de constitution et de transmission de connaissances, dont la diffusion est cependant contrôlée. Nous analysons dans un second temps l'évolution des vecteurs de circulation et son influence sur la discipline, en nous appuyant sur la biographie de deux mathématiciens saxons, J.A. Scheidhauer (1718-1784) et son discipline J.F. Lempe (1757-1801). Leurs parcours croisés symbolisent la transformation de la discipline. Le passage à l'imprimé, la publication d'articles dans des revues spécialisées et la création de chaires académiques font de la géométrie souterraine une science reconnue qui reste cependant en dehors du corpus de connaissances et des débats universitaires.

Abstract: Subterranean geometry is a practical mathematical science which developed in mining facilities and whose dissemination was considerably modified during the 18th century. This evolution was related to the gradual institutionalization of the discipline - from the creation of a companionship system to the foundation of mining academies. Practices gradually incorporated new methods and came to be based on solid basic training in theoretical mathematics. The dissemination of knowledge and teaching were initially based on a system of manuscripts, which will be analyzed in detail. The example of 
the Markscheider August Beyer (1677-1753) illustrates this collective process of creating and transmitting knowledge, the dissemination of which was nevertheless controlled. We shall then analyze the evolution of vectors for dissemination and how this influenced subterranean geometry by reconstructing the biography of two Saxon mathematicians, J.A. Scheidhauer (1718-1784) and his follower J.F. Lempe (1757-1801). Their interlinked approaches illustrate the transformation of the discipline. The move to printing as well as the publication of articles in specialized journals and the creation of academic chairs turned subterranean geometry into a recognized science but one which nevertheless remained outside the main corpus of university knowledge and its debates.

\section{Introduction}

Dans les régions montagneuses d'Europe centrale, l'usage de la géométrie pratique est nécessaire au bon fonctionnement des mines. À partir du XVI ${ }^{\mathrm{e}}$ siècle, il est fréquemment fait mention de géomètres souterrains : il s'agit d'une profession différenciée, qui possède des compétences techniques et scientifiques particulières. Ces régions forment des îlots géographiquement isolés qui possèdent une culture commune, entre lesquels circulent hommes et connaissances ${ }^{1}$. Confrontés à des problèmes similaires, ils développent aux XVII ${ }^{\mathrm{e}}$ et XVIII ${ }^{\mathrm{e}}$ siècles un ensemble de savoirs, les sciences des montagnes, parmi lesquelles figurent en bonne place la géométrie souterraine [en allemand, Markscheidekunst, en latin geometria subterranea].

Cette discipline constitue à l'origine un ensemble de connaissances et de techniques éminemment pratiques développées dans un but concret : mettre des savoirs géométriques au service de l'exploitation minière. Le géomètre souterrain est membre de l'administration des mines. Il doit à ce titre établir et tenir à jour le cadastre puis établir des cartes détaillées des exploitations. Il doit également réaliser les mesures et calculs qui permettent de réaliser les percements de galeries. Son rôle s'étend enfin parfois, au cours du XVIII ${ }^{\mathrm{e}}$ siècle, à d'autres domaines des mathématiques pratiques, à l'ingénierie et à l'hydrodynamique, notamment pour assurer l'approvisionnement en eau. Transmises à un nombre croissant de personnes, ces connaissances sont perfectionnées par des générations successives de géomètres.

Ces processus ont cependant été très peu étudiés, et l'on ne sait pratiquement rien des échanges et de l'enseignement des connaissances avant la création d'académies des mines dans l'espace germanophone. Cet article apporte une contribution à l'histoire de la géométrie souterraine, considérée en tant que science mathématique pratique. À cet égard, nous montrerons qu'il

1. Voir par exemple [Hillegeist \& Liessmann 2001]. 
s'agit d'un véritable microcosme, aux deux sens du terme. Le milieu de la géométrie souterraine est tout d'abord un petit monde dans lequel les savoirs et même les ouvrages s'élaborent de manière largement collective. À l'intérieur du cercle des praticiens, les manuscrits circulent efficacement selon des règles précises. La riche tradition intellectuelle des régions minières d'Europe centrale, culturellement et géographiquement circonscrites, a une influence profonde sur l'organisation de ces échanges. Mais la géométrie souterraine est aussi un microcosme au sens où elle constitue un milieu particulier, avec ses propres méthodes et problématiques. L'ouverture progressive qui accompagne son institutionnalisation est un exemple de l'évolution des mathématiques pratiques au cours du XVIII ${ }^{\mathrm{e}}$ siècle.

Nous décrirons dans une première partie la culture des manuscrits en géométrie souterraine et son fonctionnement à l'aide d'exemples principalement tirés de la région des Monts Métallifères de Saxe. Nous montrerons que la formation des géomètres souterrains, l'établissement et la diffusion des connaissances possèdent leur propre organisation et sont conçus comme une entreprise collective. La seconde partie se focalise sur le dernier tiers du XVIII ${ }^{\mathrm{e}}$ siècle, au cours duquel l'institutionnalisation et la mathématisation des pratiques entraînent une modification profonde des vecteurs d'échanges. Deux mathématiciens peu connus nous permettront d'illustrer ce processus. Le directeur des mines J.A. Scheidhauer, autodidacte, est l'auteur de nombreux manuscrits dont les méthodes novatrices sont restées largement confidentielles. Son disciple J.F. Lempe, professeur de mathématiques à l'Académie des mines, s'attache au contraire à largement diffuser et mettre en pratique les résultats des travaux menés collectivement à Freiberg.

\section{Diffuser sans imprimer : la culture manuscrite en géométrie souterraine}

\subsection{Manuscrits et formation des géomètres souterrains}

Les Monts Métallifères sont une région montagneuse située au Sud de l'Électorat de Saxe, qui a historiquement joué un rôle important dans l'essor des sciences des montagnes en Europe ${ }^{2}$. Sa ville principale, Freiberg, est peu éloignée de la capitale de l'État, Dresde, ainsi que de Leipzig, qui accueille l'une des plus grandes universités allemandes et est renommée pour sa foire du livre bisannuelle, qui réunit l'essentiel des éditeurs germanophones. Dans les Monts Métallifères, les géomètres souterrains [Markscheider] sont évalués et

2. Sur l'histoire culturelle de la Saxe, on consultera avec profit [Espagne 2000]. Sur le terme de sciences des montagnes [Bergbaukunde] et ses spécificités, voir [Laboulais $2012,14-15]$. 
assermentés par un conseil des mines [Oberbergamt] situé à Freiberg. Un géomètre travaille sur l'ensemble des exploitations dans un territoire géographique précis. Il doit visiter chaque galerie et procéder à des mesures à l'aide des instruments usuels : la boussole suspendue [Hängekompass], le fil à plomb et le niveau [Wasser-Waage]. Ces mesures lui permettent non seulement de cartographier les mines - ce qui est exigé par les actionnaires [Kuxen-Inhaber] et par l'administration des mines -, mais également de diriger les coûteux percements de galeries. Il est enfin chargé de faire respecter les limites de propriétés et de régler les cas litigieux lorsque deux galeries se rencontrent sous terre.

Dans la première moitié du XVIII ${ }^{\mathrm{e}}$ siècle, il n'existe pas d'établissement qui assure la formation des géomètres souterrains et la circulation des connaissances. Malgré sa puissance économique et une population d'environ 10000 habitants, Freiberg reste située en périphérie des centres littéraires et intellectuels allemands. Elle n'est pas encore le haut lieu de l'Aufklärung qu'elle deviendra après la création de l'Académie des mines ${ }^{3}$. L'unique lycée [Gymnasium] prépare à l'université de Leipzig, et fait ainsi la part belle à la religion et aux langues classiques. La direction des mines et des fonderies nécessite cependant des compétences techniques et une formation pratique. Un système de compagnonnage existe dès le XVII ${ }^{\mathrm{e}}$ siècle, est officialisé en 1702 et est dès lors soutenu par le prince-électeur de Saxe. Des bourses sont distribuées afin de permettre à de jeunes Saxons de suivre auprès de certains fonctionnaires des mines un entraînement pratique, cf. [Sennewald 2002]. Cet enseignement concerne exclusivement la docimasie (essayage des métaux) et la géométrie souterraine.

Chaque année, deux ou trois personnes apprennent ainsi le maniement des instruments et les tâches du métier de Markscheider auprès d'un géomètre assermenté par l'administration ${ }^{4}$. Dans la première moitié du siècle, il s'agit le plus souvent d'August Beyer (1677-1753), nommé géomètre souterrain à Freiberg en 1697 et conseiller du prince-électeur de Saxe $^{5}$. Cet apprentissage est sanctionné par un certificat, qui garantit les aptitudes des futurs géomètres. Un premier avantage de ce système est qu'il permet la transmission de compétences directement utilisables. En 1738, Beyer délivre par exemple un certificat à un étudiant nommé Gregorius Israel Bergen, et précise que celui-ci :

[... a appris au mieux et pratiqué la science susmentionnée ou géométrie souterraine, [c.à.d] à mesurer les travaux des mines et à les porter sur papier, à tracer là-dessus les crevasses et les filons, mais également à marquer au jour les points les plus remarquables, à indiquer la hauteur des uns par rapport aux autres aussi bien dans

3. La ville possède une imprimerie, une librairie à partir de 1746 , mais pas de bibliothèque. Voir [Schellhas 1984, 8].

4. Voir Universitätsarchiv Freiberg (dans la suite du texte UAF) - OBA 6, ff. 244r-245r, pour la liste des bourses délivrées de 1702 à 1756.

5. D'autres enseignants en géométrie souterraine sont G. G. Seybt, Vizemarkscheider, puis C.E. Richter, Markscheider et Bergamtassessor. Voir [Sennewald 2002, 418]. 
un plan géométral [Grund Riß] que dans le plan de coupe [Profil oder Seÿger-Riß], y ajouter les bâtiments de surface, les puits environnants et les bornes de concessions, la manière dont tous les puits et galeries sont mesurés par l'arithmétique, les percements dirigés par la géométrie, afin que l'on puisse s'enquérir de la structure de toute la mine, y niveler les conduits et les profondeurs, et qu'il s'est si bien qualifié dans cet art et cette science, qu'il est en tout point un bon géomètre souterrain et pourra assurer un bon service. [UAF - OBA 5, ff. 142v-143r, notre traduction (comme pour l'ensemble de l'article, sauf indication contraire)]

Un second avantage est que la circulation de ces connaissances est ainsi, sinon confidentielle, du moins contrôlée. Ces enseignements ont en effet lieu pour partie dans les mines, ainsi que chez les géomètres. Ils utilisent des manuscrits, comme le Nouveau Livre de géométrie souterraine (1669), dans lequel Adam Schneider (1634-1707) décrit par exemple l'usage des tables trigonométriques et de la boussole suspendue ${ }^{6}$. Ces manuscrits ne sont pas destinés à être imprimés et leur diffusion est au contraire soigneusement encadrée. Chaque géomètre souterrain possède une copie personnelle, qui est dictée ou parfois prêtée aux étudiants. Ceux-ci sont autorisés à en faire une copie, mais doivent ensuite " garder la main dessus, et ne la rendre publique ou ne la brader à personne, pour de l'argent ou pour toute autre raison ${ }^{7} »$.

\subsection{Contexte d'utilisation des connaissances mathématiques pratiques}

La diffusion des connaissances est ainsi assurée à l'intérieur d'un système $\operatorname{clos}^{8}$. $\mathrm{Au}$ début $\mathrm{du} \mathrm{XVIII}^{\mathrm{e}}$ siècle, la géométrie souterraine est une science ésotérique au sens où elle est pratiquée par des individus initiés dans un but bien spécifique, celui de l'exploitation des mines. De plus, elle fait largement appel à des concepts et à un vocabulaire spécifique (la Bergmannsprache, ou « langue des mineurs $\left.{ }^{9} »\right)$. On apprend avec un géomètre souterrain, qui enseigne non seulement des connaissances technico-mathématiques, mais aussi un vocabulaire particulier et surtout la mise en pratique et l'utilisation des instruments

6. Le manuscrit se trouve à la TU Bergakademie Freiberg, Universitätsbibliothek (dans la suite du texte TU BAF-UB), XVII 18. Sur la biographie d'A. Schneider, voir [Riedel 2006].

7. Règlement établi à Schemnitz, alors en Basse-Hongrie, 22 juin 1735 [Schmidt 1834, 481].

8. Dans les faits, certain manuscrits seront imprimés, comme en témoigne par exemple la publication en 1686, par N. Voigtel, d'une Geometria Subterranea [Voigtel 1686] qui emprunte au manuscrit de son maître A. Schneider.

9. J.-P.-F. Duhamel, dans son dictionnaire des mines allemand-francais, insiste sur la difficulté à « rendre en français des termes que les Allemands même ne comprennent guère, s'ils n'ont pas fréquenté les ouvriers des mines » [Duhamel 1800, Introduction]. 
et méthodes dans les galeries. Le manuscrit est ainsi pleinement intégré à un contexte plus général d'enseignement et d'application. Au-delà de la protection des connaissances, la spécificité de son utilisation et la particularité de l'apprentissage réduisent la nécessité du passage à l'imprimé ${ }^{10}$.

Comme ils jouent un rôle similaire dans un contexte d'utilisation particulier, les différents manuscrits de géométrie souterraine possèdent des caractéristiques communes. On y trouve tout d'abord des listes de problèmes [Propositionen], qui correspondent aux tâches concrètes qui attendent le géomètre souterrain. Dans le manuscrit d'A. Schneider, on commence ainsi par apprendre à « diriger l'extrémité d'une galerie vers un puits de mine, et dans le même temps marquer au jour l'extrémité de la galerie ${ }^{11} »$. Leur résolution est ensuite détaillée avec des explications sur la marche à suivre, le fonctionnement des instruments à utiliser et les calculs rencontrés. Ces manuscrits ne contiennent pas de démonstrations, mais de nombreuses illustrations commentées qui les rendent directement utilisables dans la pratique. La forme du texte scientifique s'adapte ici à deux séries de contraintes inhérentes à la discipline. Elle tient compte des conditions d'enseignement et de l'aspect pratique des connaissances utilisées, tout en permettant d'en encadrer la diffusion.

August Beyer, le géomètre souterrain de Freiberg, rédige en 1708 un manuscrit intitulé Geometria Subterranea oder Markscheidekunst, qu'il utilise à la fois pour son métier et en tant qu'enseignant ${ }^{12}$. Quatre décennies plus tard, il publie un manuel sur le même sujet, basé sur ce manuscrit, sous le titre Instruction approfondie sur la science des mines, d'après les principes de la géométrie souterraine [Beyer 1749]. Il est intéressant de suivre le tortueux processus qui l'a amené à passer d'un texte à l'autre :

$\mathrm{Au}$ cours de cette longue période, il ne pouvait manquer que bien des choses nouvelles se présentent, de sorte que je dus changer ou bien remplacer, et de cette manière ce texte est passé entre les mains de plusieurs centaines de personnes, et toujours sous des formes différentes. J'ai aussi appris que certains ont modifié le sens de ce texte et l'ont ensuite fait circuler comme leur propre travail. [...] Je me suis donc décidé, il y a quelques années, à refondre complètement cette esquisse, à la modifier et à la compléter, à destination des étudiants, moyennant quoi cet ouvrage a grandi et est ainsi conçu qu'il n'a plus grand chose de semblable avec la première esquisse. [Beyer 1749, Introduction]

L'auteur nous donne ici un témoignage de première main sur le rôle des manuscrits de géométrie souterraine. Il y a bien une large diffusion au sein

10. En cela, la culture des manuscrits chez les géomètres souterrains étend l'analyse développée par P.O. Long pour le $\mathrm{XVI}^{\mathrm{e}}$ siècle. Selon elle, l'imprimé ne remplace pas le manuscrit, qui continue à jouer un rôle dans des contextes sociaux et économiques particuliers. Voir [Long 2004, 175-191].

11. TU BAF-UB, XVII 18, Neu Marckscheide-Buch, prop. 2.

12. Le manuscrit se trouve à la TU BAF-UB, XVII 12. 
de la communauté, et Beyer semble même indiquer une interaction entre auteurs et lecteurs. Les manuscrits sont ainsi en perpétuelle évolution, ce qui est accrédité par les nombreux ajouts et corrections qu'ils comportent. Cette circulation dynamique dans un groupe restreint contraste avec la quasi-absence de publication des connaissances. Beyer ne se décide à imprimer son manuscrit qu'à l'extrême fin de sa carrière, et principalement pour revendiquer la paternité du texte. Ce passage du manuscrit à l'imprimé n'est pas neutre vis-à-vis du texte et pousse Beyer à adapter aussi bien la présentation que le contenu proprement dit à un public plus large.

De fait, les manuscrits réservés au cercle étroit des géomètres sont bien différents des quelques manuels de géométrie souterraine publiés avant la création des académies des mines. Les manuscrits se focalisent sur des problèmes concrets, sur les conditions pratiques de collecte de données et sur les obligations du géomètre souterrain. Comparons les travaux d'A. Beyer avec les Institutiones Geometriae Subterraneae, ouvrage rédigé en latin et publié en 1726 par Johann Friedrich Weidler, professeur de mathématiques à l'université de Wittemberg. Cet ouvrage possède la structure d'un manuel de mathématiques classique. On y trouve un grand nombre de définitions, accompagnées de remarques [scholies], tandis que la part consacrée aux problèmes et aux instruments est réduite. Mais ce type d'ouvrage imprimé, d'autant plus lorsqu'il est rédigé en latin, est inadapté pour apprendre les opérations pratiques du métier de géomètre souterrain, qui incluent une part importante de connaissances tacites et de savoir-faire ${ }^{13}$. Après avoir constaté qu'il existe une littérature et des échanges vivaces en géométrie souterraine avant la création d'établissements d'enseignement, il faut étudier l'impact de l'institutionnalisation sur cette discipline.

\subsection{Travail collectif et institutionnalisation de l'enseignement}

Une Académie des mines [Bergakademie] est fondée en 1765 à Freiberg, dans le cadre de la réorganisation de l'État opérée après la guerre de Sept Ans (17561763) cf. [Collectif 1965]. Elle prolonge et va progressivement remplacer le système de bourses en vigueur depuis le début du siècle. Signe de l'importance des mathématiques, le professeur J.F.W. Charpentier (1738-1805) obtient la première chaire fondée à l'Académie, la seconde étant consacrée à la chimie et à la métallurgie ${ }^{14}$.

La Bergakademie offre un cursus coordonné et obligatoire en mathématiques. Charpentier, ancien étudiant de l'université de Leipzig, propose un cours de mathématiques pures basé sur les Éléments de mathématiques de

13. Sur la notion de tacit knowledge dans les sciences des mines, on consultera avec profit [Dym 2008].

14. Sur l'histoire des mathématiques à l'Académie des mines de Freiberg de sa création jusqu'en 1850, voir [Morel 2013, chap. 2, 141-252]. 
C. Wolff, suivi d'un cours de mathématiques appliquées comprenant mécanique, aérométrie, hydrostatique et hydraulique, toujours sur un modèle universitaire $^{15}$. Ces cours forment un socle commun pour aborder l'enseignement de la géométrie souterraine ${ }^{16}$. Cependant, le cours de géométrie souterraine reste confié à un géomètre souterrain, dans un premier temps Carl Ernst Richter (?-1779). Afin de contrôler la diffusion des connaissances, il n'est enseigné qu'à un petit nombre d'étudiants, dans un premier temps trois par an. L'enseignement pratique semble avoir toujours lieu de la même manière, et est toujours sanctionné par un certificat. En janvier 1781, un voyageur de passage explique que la plupart des cours, comme la minéralogie et la chimie métallique, font appel à des manuels publiés par les professeurs de l'Académie; « la géométrie souterraine est par contre enseignée d'après un manuscrit, qui indique, paraît-il, des méthodes particulières »[Beck 1781, 232 $]^{17}$.

Il est par ailleurs bien spécifié que ce cours ne doit pas se limiter « à une simple instruction théorique », mais inclure non seulement « la résolution des problèmes de géométrie souterraine, l'appréciation des instruments nécessaires et la manipulation de ces derniers (pour les relevés et le tracé), mais aussi l'exercice de ces opérations, non seulement en amenant régulièrement les élèves dans les mines, mais aussi en leur faisant effectuer et corriger plusieurs relevés [de mesures] »[UAF - OBA 10, f. 172v]. Si les formations théorique et pratique ne sont pas encore coordonnées, il faut souligner le progrès considérable en termes de formation initiale. Jusqu'en 1765, la géométrie souterraine était enseignée à des élèves ayant des connaissances très hétérogènes et souvent réduites. Un premier mérite des académies des mines est donc de fournir un enseignement théorique en géométrie élémentaire, nécessaire pour le développement ultérieur des sciences des montagnes.

À l'Académie des mines de Freiberg, la géométrie souterraine est de plus pratiquée comme une science collective. On peut s'en convaincre en étudiant la bibliothèque de l'établissement. C'est alors la seule de la ville, et son rôle est d'autant plus important que les livres de géométrie pratique et de sciences des montagnes, volumineux et riches en illustrations, sont onéreux. Dirigée par Charpentier, elle est librement accessible aux étudiants et semble constituer un lieu ouvert où des ressources rares sont mises en commun. Un voyageur la décrit en 1782 :

La bibliothèque de cet endroit n'est certes pas grande, mais elle possède une collection recherchée d'ouvrages mathématiques et physiques, ainsi que d'autres livres plus ou moins reliés aux sciences minières. [Hollenberg 1782, 213]

15. Son plan d'enseignement est détaillé dans un Avertissement imprimé daté du 27 avril 1767, voir UAF - OBA 236, ff.115-122.

16. À partir de 1776, un enseignement préparatoire à la Bergakademie se met en place dans les Monts Métallifères, sous la forme d'un réseau d'écoles de calcul et de dessin dans les principales villes et d'une école des mines [Bergschule] à Freiberg. Voir [Kaden 2012].

17. D. Beck précise bien qu'il n'a pas pu avoir accès à ce manuscrit. 
Elle contient non seulement l'ensemble des ouvrages imprimés qui existent en géométrie souterraine, comme celui d'A. Beyer, mais également bon nombre de manuscrits qui seraient sans cela inaccessibles. Dans les années 1780, on y ajoutera progressivement les exercices réalisés par les étudiants dans les années précédentes. Le minéralogiste A.G. Werner (c. 1749-1817), professeur à l'Académie à partir de 1775 , met lui aussi à la disposition des étudiants sa riche bibliothèque, qui contient plusieurs manuscrits de géométrie souterraine comme les Propositions de la Géometrie sous terre de J.E. Schindler ou la Geometriae, vom Markscheiden und Feldmessen de S. Span ${ }^{18}$.

Un autre aspect du caractère collectif du travail de l'Académie se trouve dans son activité éditoriale. Celle-ci couvre l'ensemble des domaines composant les sciences minières, et s'inscrit dans un temps long puisqu'elle intègre les travaux des savants des Monts Métallifères depuis le début du siècle. Il n'est pas rare qu'un ouvrage soit le résultat des efforts successifs de plusieurs personnes, dont les travaux se complètent peu à peu. C'est par exemple le cas du Traité de l'exploitation de mines [Bericht vom Bergbau], publié en 1772, et dont la page de titre n'indique pas l'auteur, mais présente symboliquement une gravure représentant l'Académie des mines de Freiberg ${ }^{19}$. Un premier manuscrit sur ce sujet à été rédigé en 1740 par Johann Gottlieb Kern, fonctionnaire des mines à Freiberg, qui s'en servait comme manuel dans ses cours particuliers. Lors de la création de l'Académie en 1765 , le capitaine général des mines et mathématicien F.W. von Oppel (c. 1720-1769) entreprend d'en éditer une version révisée pour servir de manuel, mais son décès soudain interrompt l'entreprise. Le travail est finalement terminé par les autres professeurs et l'ouvrage est imprimé aux frais de l'Académie [UAF - OBA 237, 262r-262v]. Il sert non seulement de support de cours, mais également de promotion pour l'institution considérée comme entité, d'autant plus qu'il s'ouvre sur un Avertissement détaillant l'organisation et le programme de l'Académie.

Focalisons-nous à présent sur l'évolution des vecteurs d'échanges durant le dernier tiers du XVIII ${ }^{\mathrm{e}}$ siècle, période charnière pour l'institutionnalisation de la géométrie souterraine. Il faut en particulier chercher à préciser les liens entre les pratiques antérieures fondées sur la circulation de manuscrits et l'enseignement académique, ouvert et largement diffusé en Europe. Les contributions de J.A. Scheidhauer et J.F. Lempe sont de ce point de vue particulièrement éclairantes.

18. Respectivement TU BAF-UBXVII, 17 et 14 . À sa mort, Werner lègue cette bibliothèque à l'Académie, voir [Schellhas 1969, 10-14, 20, 25]. Elle contient alors près de 14000 volumes, aujourd'hui identifiables grâce à son ex-libris.

19. [Oppel \& Kern 1772]. Le bâtiment de l'Académie des mines est la maison de F.W. von Oppel, qui l'a léguée à sa mort en 1769. Il a également cédé pour une somme symbolique sa collection d'instruments et sa bibliothèque, représentées sur la gravure. 


\section{3 Évolutions de la discipline à l'Académie des mines de Freiberg}

L'enseignement de la géométrie souterraine à l'Académie des mines prend un nouvel essor dans les années 1780, sous l'impulsion de Johann Friedrich Lempe (1757-1801), qui s'efforce de faire dialoguer théorie et pratique pour renforcer l'utilité [Brauchbarkeit] des mathématiques dans l'exploitation des mines. Ancien étudiant de l'Académie, Lempe a pu y étudier la géométrie souterraine et profiter des manuscrits les plus récents, en particulier ceux de J.A. Scheidhauer ${ }^{20}$. Il commence par enseigner les mathématiques à l'École des mines locale et remplace progressivement J.F.W. Charpentier à l'Académie. Pour apprécier l'influence de Lempe sur l'évolution de la géométrie souterraine, il faut introduire les travaux de son mentor J.A. Scheidhauer.

\subsection{La mathématisation des pratiques dans les manuscrits de J.A. Scheidhauer}

Johann Andreas Scheidhauer est né en 1718 à Johanngeorgenstadt, petite ville minière située au cœur des Monts Métallifères. Malgré ses importantes contributions au développement de la géométrie souterraine, il n'a jamais fait l'objet d'une biographie ${ }^{21}$. Son père, J.A. Scheidhauer senior (1675-1753) occupe une position intermédiaire dans l'administration locale des mines et peut donc envoyer son fils étudier à l'université de Leipzig. Il y étudie probablement le droit et suit également quelques cours de physique et de mathématiques. Il devient en 1743 assistant greffier des mines [Vize-Bergschreiber] auprès de son père, auquel il succède dix ans plus tard.

C'est sans doute à cette époque qu'il apprend la géométrie souterraine bien que ce ne soit pas son métier - au contact des Markscheider de Johanngeorgenstadt ${ }^{22}$. Il se forme aussi en mathématiques théoriques, ce qui est un défi dans une petite ville de montagne isolée du monde extérieur plu-

20. Il a également étudié à l'université de Leipzig avec le mathématicien Carl Friedrich Hindenburg (1741-1808). Sur la biographie de J.F. Lempe, voir TU BAFUB, Dieter Flaxa, Studie über Johann Friedrich Lempe, Januar 1985, ainsi que [Kaden 2013]

21. Les paragraphes suivants constituent ainsi une courte esquisse biographique, principalement réalisée à partir de son Nachlass [TU BAF-UB Nachlass 115] et divers documents issus des archives locales.

22. Parmi les manuscrits qui circulent probablement à cette époque dans la région de Johanngeorgenstadt, on trouve le De Geometria Subterranea, Gründliche und aus erfahrenheit stammende Marckscheide-Kunst, rédigé de 1693 à 1696 par le Markscheider local, Johann Scherez. 
sieurs mois par an et ne disposant d'aucune librairie ${ }^{23}$. J.A. Scheidhauer est nommé en 1765 à la direction locale des mines [Bergmeister] à Freiberg, sans cependant officiellement enseigner à la Bergakademie. En plus de ses responsabilités administratives, il cherche à utiliser les mathématiques dans l'exploitation minière. Il a rédigé plus d'une centaine de volumes manuscrits, pour la plupart écrits en allemand, occasionnellement en latin et plus rarement en français. Ils abordent des sujets variés, qui vont de l'étude des roues à aubes à des relevés de profondeurs, en passant par la mise au point d'un système de mesure de longueur unifié dans les Monts Métallifères.

Il apporte surtout de nombreuses contributions théoriques en géométrie souterraine, consignées sous la forme de collections d'exercices. Il rédige également un manuel, intitulé Contributions à la géométrie souterraine, dont « le but principal [...] est de résoudre par le calcul toutes les instructions qui peuvent être exigées d'un géomètre souterrain ${ }^{24} »$. Dans ses manuscrits, il suppose connus du lecteur non seulement les mathématiques élémentaires, mais également l'ensemble du manuel d'A. Beyer (version de 1749), et se place ainsi en continuateur de ce dernier. Il s'attache à éviter les résolutions par le dessin pour ne proposer que des solutions calculatoires; les démonstrations sont rares, mais l'on trouve de multiples exemples.

Scheidhauer est membre de la Société économique de Leipzig [Leipziger ökonomische Sozietät] fondée en $1764^{25}$ et se rend fréquemment à la foire du livre. Nous pensons qu'il a mis à profit ses voyages pour acquérir la littérature qui lui manque, par exemple les Comptes rendus de l'Académie des sciences de Berlin. Cette hypothèse est corroborée par ses nombreuses copies d'articles et d'ouvrages de mathématiciens contemporains, comme L. Euler, A.-C. Clairaut, J.H. Lambert ou d'Alembert. La Société sollicite en 1767, puis à nouveau en 1770 , des contributions de sa part ${ }^{26}$. Scheidhauer finit par obtempérer et publie finalement ses deux seuls articles jamais imprimés dans les Annonces de la Société pour la foire de Leipzig 1772, soit une trentaine de pages dans une publication relativement confidentielle. Le premier concerne la mesure de la déclinaison magnétique, et le second la détermination à partir de trois points de la direction principale et de l'inclinaison d'un filon de minerai [Scheidhauer 1772a,b].

Ce second article (voir Figure 1) correspond à une tâche typique en géométrie souterraine, que l'on trouvait par exemple dans le manuscrit de $\operatorname{Beyer}^{27}$.

23. On trouve dans son Nachlass non seulement des copies manuscrites de manuels de mathématiques élémentaires, mais aussi par exemple des Instituzioni Analitiche de M.G. Agnesi [TU BAF-UB Nachlass 115, 300d, vol. 1].

24. TUf-WA Nachlass 115, 300m, Beyträge zur Markscheidekunst, avant-propos non paginé.

25. Sur la Société économique de Leipzig voir [Schöne 2001].

26. Voir TU BAF-UB Nachlass Scheidhauer, $300 \mathrm{u}+$ sonstiges, lettre du 20 octobre 1767 et Pro memoria du 5 novembre 1770.

27. Voir TU BAF-UB, XVII 12, Geometria Subterranea de Beyer, en particulier propositions 9,16 et 49 . 


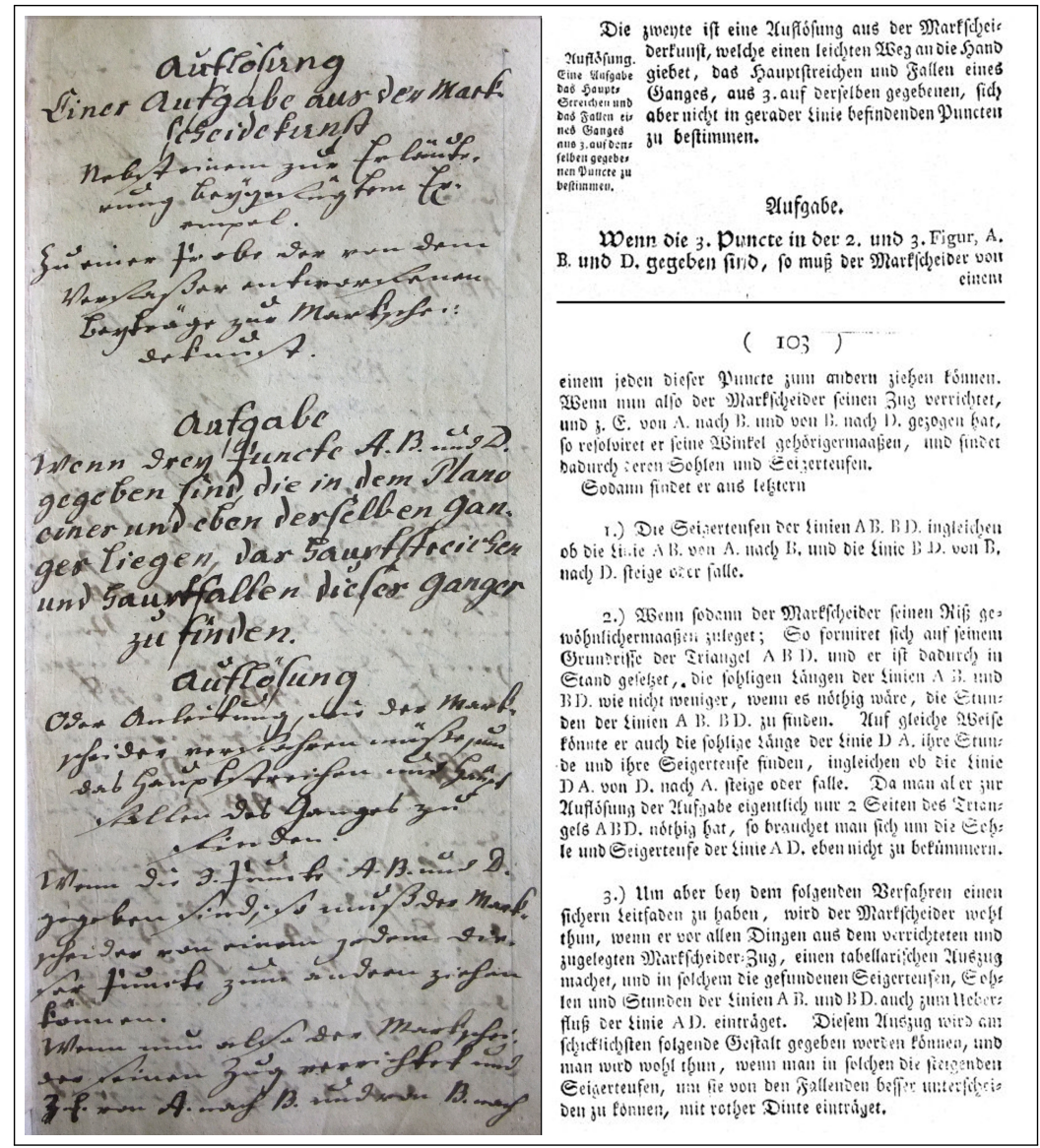

FiguRE 1 - Manuscrit et version imprimée de l'article de J.A. Scheidhauer sur la détermination à partir de trois points de la direction principale [Hauptstreichen] et de l'inclinaison [Hauptfallen] d'un filon de minerai (source : TU BAF-UB Nachlass Scheidhauer, 300m et [Scheidhauer 1772a, 102-103]).

Scheidhauer y apporte plusieurs innovations. D'une part, il donne une méthode générale, là où Beyer considérait de multiples cas particuliers selon l'orientation des filons, certains cas n'étant pas traités. D'autre part, il expose la méthode traditionnelle, dans laquelle les mesures sont portées sur la carte et le problème est ensuite résolu en utilisant le rapporteur et des méthodes graphiques. Il donne cependant une seconde méthode, purement calculatoire, et souligne que le résultat « est déterminé de manière bien plus sûre et fiable [viel richtiger und zuverlässiger] par le calcul à partir des angles trouvés par le géomètre 
pendant son relevé » [Scheidhauer 1772a, 109]. Cet exemple illustre la volonté de Scheidhauer d'uniformiser les pratiques et d'introduire autant que possible des résolutions calculatoires et analytiques.

\section{$3.2 \quad$ J.F. Lempe et l'évolution graduelle des vecteurs de circulation}

Il est difficile de répondre avec certitude à la question du public des manuscrits de J.A. Scheidhauer, dont plusieurs s'adressent explicitement à un lecteur. Il est cependant clair qu'ils ont eu une certaine circulation dans les Monts Métallifères et que les étudiants les plus doués de l'Académie y ont eu accès, au premier rang desquels J.F. Lempe, qui commence ses études à Freiberg en 1773. On voit donc une fois de plus que les documents manuscrits continuent de jouer un rôle important après la création de l'Académie des mines. Au début des années 1780, J.F. Lempe va s'efforcer de publier ces documents, afin d'accélérer la diffusion des connaissances et l'adoption de méthodes plus mathématisées. Il publie ainsi dans le Leipziger Magazin zur Naturkunde, Mathematik und Oekonomie de C.F. Hindenburg, un article intitulé « Nouvelle méthode pour trouver la direction principale d'un filon », qui se base directement sur l'article de Scheidhauer de $1772^{28}$. En 1781 et 1782 , il publie ensuite deux manuels qui vont faire connaître à un large public les travaux de Scheidhauer. Il souligne à de nombreuses reprises les emprunts qu'il a faits et considère son propre travail comme la poursuite de celui de son mentor :

Du reste, Monsieur le directeur des mines Scheidhauer a rédigé un manuscrit dans lequel des solutions complètes, la plupart cependant sans démonstration, sont données à la plupart des problèmes qui peuvent se poser en géométrie souterraine, parfois accompagnées d'illustrations.

Ce manuscrit contient, comme annoncé, de très précieuses contributions à la géométrie souterraine, qui si elles étaient imprimées seraient plus utiles au public, et ne pourraient être que bienvenues pour chaque géomètre souterrain qui cherche à exercer correctement son art. [Lempe 1782, 178-179]

Chargé des cours de mathématiques de l'Académie à partir de 1783, Lempe enseigne bientôt la partie théorique de la discipline aux étudiants, tout d'abord dans le cadre du cours de mathématiques pures ${ }^{29}$. À partir de 1795 , un nouveau cours de "géométrie souterraine théorique » est introduit, et les étudiants continuent à suivre des cours pratiques dans les mines. Les aspects théoriques

28. [Lempe 1781]. En 1783, il publie un second article en géométrie souterraine [Lempe 1783].

29. Voir par exemple la collection d'exercices proposés par J.F. Lempe en 1784, en particulier les problèmes 51 à 71, dans UAF - OBA 245, ff. 135r-161r. 
et pratiques sont étroitement coordonnés afin de maximiser l'utilité de la formation. Le cours de Lempe s'appuie sur un ouvrage qui présente lui aussi toutes les caractéristiques d'un travail collectif dans le temps. Il s'agit d'une réédition, considérablement retravaillée par Lempe, du manuel de géométrie souterraine de Beyer qui était devenu introuvable. Elle paraît en 1775, trois quarts de siècle après la première esquisse de Beyer ${ }^{30}$ et compte 1176 pages, soit près de cinq fois plus que la première édition de 1749. Lempe indique en outre avoir emprunté à plusieurs géomètres et fonctionnaires de Freiberg, au premier rang desquels ont trouve Scheidhauer, leurs nouvelles méthodes, avant de les formaliser et de les mathématiser, afin de fournir un ouvrage exhaustif aussi utile que possible dans la pratique.

Après avoir connu jusqu'au milieu du XVIII ${ }^{\mathrm{e}}$ siècle un développement essentiellement confidentiel, la géométrie souterraine fait donc l'objet à Freiberg de nombreuses publications imprimées au début des années 1780, phénomène qui s'étend bientôt aux autres régions minières germanophones. Ces publications font avant tout suite à l'institutionnalisation de la formation des géomètres et à la fondation de plusieurs académies des mines. Outre l'Académie des mines de Freiberg en Saxe, l'Autriche-Hongrie s'est en effet dotée en 1770 d'une Académie des mines implantée à Schemnitz. La même année, une Académie des mines est fondée à Berlin, capitale de la Prusse, tandis qu'en 1775 une école des mines est ouverte à Clausthal, dans l'Électorat de Hanovre ${ }^{31}$. Les ouvrages, articles et manuels imprimés par les professeurs et administrateurs de ces régions reprennent, collationnent et améliorent des manuscrits précieusement conservés jusqu'alors. Dans ces académies, on trouve donc à la fois de nouvelles chaires de mathématiques pratiques et un public plus large pour la géométrie souterraine. Des enseignements autrefois protégés sont progressivement ouverts aux étudiants étrangers. L'institutionnalisation de l'enseignement et le passage à l'imprimé constituent deux facteurs majeurs de l'évolution des vecteurs d'échanges.

\subsection{Circulation des publications en géométrie souterraine}

Malgré tout, la diffusion de la géométrie souterraine emprunte toujours à la fin $\mathrm{du} \mathrm{XVIII}^{\mathrm{e}}$ siècle des canaux bien spécifiques. Comme le révèle une analyse des publications imprimées, elle reste le plus souvent en marge de la littérature mathématique de l'époque. Remarquons tout d'abord que la géométrie souterraine est peu ou pas représentée dans les milieux universitaires germanophones. À quelques exceptions près, les manuels utilisés dans les universités n'abordent pas le sujet, en dépit de son intérêt économique évident, alors que

30. [Beyer \& Lempe 1785], voir ci-dessus p. 22 pour le passage du manuscrit de 1708 à l'édition de 1749 .

31. Sur Berlin, voir [Klein 2010], sur Schemnitz [Morovics 2010]. 
le large spectre des disciplines mathématiques représentées inclut par exemple la géographie, l'aérométrie, la gnomonique et même le calcul marchand ${ }^{32}$.

Où sont donc publiés les travaux de géométrie souterraine? Outre les manuels rédigés par les professeurs de mathématiques des académies, on trouve de nombreux articles de journaux. La discipline profite ainsi de l'essor des périodiques spécialisés dans l'espace germanophone dans le dernier quart du siècle, cf. [Morel \& Bullynck 2015, en particulier fig. 1]. Il peut s'agir de publications locales comme les Annonces semestrielles de la Société économique de Leipzig, dans lesquelles paraissent les articles de Scheidhauer. On trouve aussi des publications influencées par le mouvement caméraliste. Ignaz von Born (1742-1791), qui a étudié à l'Académie de Schemnitz, édite plusieurs volumes de mémoires consacrés aux mathématiques et à l'histoire naturelle ${ }^{33}$. De nombreux articles y sont consacrés à l'exploitation des mines, et parmi ceux-ci plusieurs à la géométrie souterraine et ses instruments. En 1805, un artisan mécanicien de Cassel publie un Magazine des dernières nouvelles mathématiques pour les ingénieurs, militaires, architectes, forestiers, géomètres souterrains et mécaniciens, lui aussi fortement orienté vers l'aspect instrumental de la discipline [Breithaupt 1805-1806].

Le principal vecteur de circulation de la géométrie souterraine est cependant incontestablement le magazine de J.F. Lempe. Celui-ci cesse rapidement de publier dans les journaux de Hindenburg, qui se spécialisent progressivement dans les parties plus théoriques des sciences mathématiques ${ }^{34}$. Il décide en 1786 de doter l'Académie des mines d'une publication périodique, à laquelle il donne le titre de Magazin für die Bergbaukunde. Il annonce clairement dans le premier numéro son orientation vers les mathématiques pratiques :

J'inclurai ici les travaux qui ont été réalisés, à mon instigation et sous ma supervision, par l'un ou l'autre de mes auditeurs; bien sûr uniquement les travaux qui ne me sembleront pas indignes d'être publiés; et ces travaux pourront être un exemple de mes efforts d'enseignement pour rendre les mathématiques aussi utiles que possible à l'exploitation des mines. [Lempe 1785-1799, vol. 1, Introduction]

De fait, ce journal est utilisé par Lempe pour diffuser ses travaux et ceux de ses étudiants dans le domaine de la géométrie souterraine et plus généralement des mathématiques pratiques. Les contributions sont parfois signées de

32. S'il faut souligner qu'A.G. Kästner a publié un manuel de géométrie souterraine (en 1775), celui-ci ne semble pas avoir été régulièrement utilisé dans un cadre universitaire. Ainsi, une étude des programmes universitaires de l'université de Leipzig jusqu'au milieu du XIX ${ }^{\mathrm{e}}$ siècle montre qu'aucun cours n'aborde la géométrie souterraine. Voir [Morel 2013, 180].

33. [Born 1775-1784]. Sur la diffusion des connaissances dans les sciences des mines, voir [Vogel 2013].

34. Sur l'histoire des journaux publiés par Hindenburg, voir par exemple [Bullynck 2013]. 
Lempe, mais le plus souvent anonymes. Il s'agit alors d'exercices proposés par le professeur à ses étudiants, qui s'élaborent une fois de plus collectivement, afin d'apporter une solution mathématique à un problème précis, cf. [Morel 2013, 169, 176]. Le choix d'utiliser les mathématiques de manière systématique pour encourager l'exploitation des mines est critiqué par certains ; le journal de Lempe devient cependant un périodique de référence et voit sa diffusion s'étendre à l'Europe entière. Il est par exemple disponible à l'École des mines de Paris et est cité dans des publications francophones.

\section{Conclusion}

Les échanges en géométrie souterraine se transforment rapidement au cours du dernier tiers du XVIII ${ }^{\mathrm{e}}$ siècle. Il s'agissait jusque-là d'un savoir précieux conservé sur manuscrits, transmis à un petit nombre d'élèves dans un contexte spécifique d'apprentissage. Quelques décennies plus tard, la discipline fait l'objet de publications imprimées de nature variée, librement diffusées dans toute l'Europe. Cette évolution des vecteurs d'échange est contemporaine de la création d'académies des mines. L'institutionnalisation n'a certes pas provoqué à elle seule la mathématisation de la discipline, déjà existante dans un petit nombre d'ouvrages antérieurs, mais contribue puissamment à la diffusion et à l'accélération du phénomène. Le passage à l'imprimé et la nécessité d'intégrer la géométrie souterraine à un cursus académique modifient la discipline en profondeur. Le vecteur d'échange transforme ici l'objet scientifique transmis, dans son organisation comme dans son contenu.

Si les problèmes pratiques abordés restent les mêmes, une attention accrue est portée à leur résolution par le calcul, tandis que des procédés analytiques remplacent progressivement les approximations et les résolutions par le dessin. Les trajectoires croisées de J.A. Scheidhauer et de son élève J.F. Lempe illustrent cette dynamique. Les travaux d'A. Beyer étaient principalement tirés de son expérience personnelle en tant que Markscheider. Ceux de J.A. Scheidhauer marquent un renouveau de la discipline, sans cependant faire évoluer le mode de diffusion des connaissances. J.F. Lempe, professeur de mathématiques à l'Académie des mines, poursuit le travail théorique de son mentor tout en changeant radicalement les vecteurs d'échange utilisés.

Cette évolution ne fait cependant pas entrer la géométrie souterraine dans le champ des mathématiques universitaires allemandes de l'époque. À la fin du XVIII ${ }^{\mathrm{e}}$ siècle, les controverses en géologie et minéralogie sont discutées par toute l'Europe savante et font de Freiberg, où enseigne A.G. Werner, un centre des Lumières allemandes. En dépit des nouveaux modes de diffusions, ce ne sera pas le cas pour la géométrie souterraine, qui reste un microcosme. Il est certes plus aisé de diffuser des connaissances, qui peuvent être publiquement discutées et améliorées. Les échanges qui existent restent néanmoins circonscrits à quelques régions, entre les géomètres souterrains des Monts Métallifères, 
du Harz et de Schemnitz, ou bien avec les écoles des mines de Paris, Berlin ou Saint-Pétersbourg. Cette science pratique est intimement liée à une langue, des problèmes et des instruments. Ces éléments ne peuvent que partiellement se transmettre par le biais de livres. Bien que diffusée dans des ouvrages et des journaux spécialisés, elle reste avant tout une entreprise collective, pratiquée et discutée dans les académies des mines.

\section{Remerciements}

Je tiens à remercier les deux rapporteurs pour leurs remarques constructives. Merci également à Jeanne Peiffer pour ses suggestions, ainsi que pour avoir accepté de relire une seconde version de cet article.

\section{Bibliographie}

BECK, Dominicus [1781], Briefe eines Reisenden von +++ an seinen guten Freund $z u+++$ über verschiedene Gegenstände der Naturlehre und Mathematik, Salzburg : Erbinn.

BEYER, August [1749], Gründlicher Unterricht von Berg-Bau nach der Anleitung zur Markscheidekunst, Schneeberg : Fulden.

Beyer, August \& Lempe, Johann Friedrich [1785], Gründlicher Unterricht vom Bergbau nach der Anleitung zur Markscheidekunst, Altenburg : Richter.

BorN, Ignaz von (éd.) [1775-1784], Abhandlungen einer Privatgesellschaft in Böhmen zur Aufnahme der Mathematik, der vaterländischen Geschichte, und der Naturgeschichte, t. 1-6, Prag : Gerle.

Breithaupt, Heinrich Carl Wilhelm (éd.) [1805-1806], Magazin für das Neueste aus der Mathematik für Ingenieur, Militair, Architekten, Forstbedienten, Markscheider und Mechaniker, t. 2, Fleischer.

Bullynck, Martin [2013], Stages towards a German mathematical journal (1750-1800), dans Les Journaux savants dans l'Europe moderne : communication et construction des savoirs, édité par J. Peiffer, M. Conforti \& P. Delpiano, Amsterdam : Brepols, Archives internationales d'histoire des sciences, t. 63, 237-251.

Collectif [1965], Bergakademie Freiberg : Festschrift zu ihrer Zweihundertjahrfeier am 13. Nov. 1965, Leipzig : Verlag für Grundstoffindustrie.

Duhamel, Jean-Pierre-François [1800], Dictionnaire portatif, allemand et français contenant les mots techniques relatifs à l'art d'exploiter les mines, Paris : Courcier. 
Dym, Warren [2008], Scholars and miners : Dowsing and the Freiberg Mining Academy, Technology and Culture, 49(4), 833-859.

Espagne, Michel [2000], Le Creuset allemand. Histoire interculturelle de la Saxe $\mathrm{XVIII}^{e}-\mathrm{XIX}^{e}$ siècles, Paris : PUF.

Hillegeist, Hans-Heinrich \& Liessmann, Wilfried (éd.) [2001], Technologietransfer und Auswanderungen im Umfeld des Harzer Montanwesens, Harz-Forschungen, t. 13, Berlin : Lukas.

HollenberG, Georg Heinrich [1782], Bemerkungen über verschiedene Gegenstände auf einer Reise durch einige deutsche Provinzen, in Briefen, Stendal : Franzen und Große.

KADEN, Herbert [2012], Das sächsische Bergschulwesen : Entstehung, Entwicklung, Epilog (1776-1924), Cologne : Böhlau.

[2013], Johann Friedrich Lempe (1757-1801) : Lehrer für "Bergpurschen" und Professor für Mathematik, Physik und Bergmaschinenwesen an der Bergakademie Freiberg, Historische Schriftenreihe des Universitätsarchivs Freiberg, t. 5, Freiberg : TU Bergakademie.

KLEIn, Ursula [2010], Ein Bergrat, zwei Minister und sechs Lehrende : Versuche der Gründung einer Bergakademie in Berlin um 1770, NTM Zeitschrift für Geschichte der Wissenschaften, Technik und Medizin, 18(4), 437-468.

Laboulais, Isabelle [2012], La Maison des mines : la genèse révolutionnaire d'un corps d'ingénieurscivils (1794-1814), Rennes : Presses universitaires de Rennes.

LEMPE, Johann Friedrich [1781], Neue Methode, das Hauptstreichen eines Ganges zu finden, Leipziger Magazin zur Naturkunde, Mathematik und Oekonomie, 1, 187-201.

—_ [1782], Gründliche Anleitung zur Markscheidekunst, Leipzig : Crusius.

[1783], Auflösung einer Aufgabe aus der Markscheidekunst, Leipziger Magazin zur Naturkunde, Mathematik und Oekonomie, 3, 177-188.

LEMPE, Johann Friedrich (éd.) [1785-1799], Magazin für die Bergbaukunde, t. 13 .

Long, Pamela O. [2004], Openness, Secrecy, Authorship, Baltimore : Johns Hopkins University Press.

Morel, Thomas [2013], Mathématiques et politique scientifique en Saxe (17651851). Institutions, acteurs et enseignements, Thèse de doctorat, université Bordeaux 1. 
Morel, Thomas \& Bullynck, Maarten [2015], Une révolution peut en cacher d'autres. Le paysage morcelé des mathématiques dans l'espace germanophone et ses reconfigurations, dans Sciences mathématiques 1750-1850: continuités et ruptures, édité par C. Gilain \& A. Guilbaud, Paris : CNRS Éditions.

Morovics, Miroslav Tibor [2010], The mathematics and its professors at the Mining and Forestry Academy in Schemnitz (Banská Štiavnica), dans Mathematics in the Austrian-Hungarian Empire. Proceedings of a Symposium held in Budapest on August 1, 2009 during the XXIII ICHST, Prague : Matfyzpress, 93-102.

OpPel, Friedrich Wilhelm von \& Kern, Johann Gottlieb [1772], Bericht vom Bergbau, Leipzig : Crusius.

RIEDEL, Lothar [2006], Adam und Johann Adam Schneider : zwei Markscheidergenerationen in Marienberg, Sächsische Heimatblätter, 52(3), 210-219.

Scheidhauer, Johann Andreas [1772a], Auflösung. Eine Aufgabe das HauptStreichen und das Fallen eines Ganges aus 3. auf denselben gegebenen Puncte zu bestimmen, Anzeige der Leipziger ökonomischen Societät in der Michaelis-Messe 1772, 17, 102-118.

[1772b], Wie die Abweichung der Magnetnadel von Norden, aus dem Aufund Untergange der Sonne durch Hülfe des Markscheider-Compasses zu bestimmen sey, Anzeige der Leipziger ökonomischen Societät in der MichaelisMesse 1772, 17, 90-102.

Schellhas, Walter [1969], Die Bibliothek der Bergakademie Freiberg 1766 1965, Freiberg : Bergakademie.

— [1984], Die Vorläufer der "Volksbibliothek für die Stadt Freiberg" : die Literaturvermittlung in Freiberg (Sachs.) von der Mitte des 18. Jahrhunderts bis 1875, Freiberg : Bergakademie.

Schmidt, Franz Anton [1834], Chronologisch-systematische Sammlung der Berggesetze der österreichischen Monarchie, vom Jahre 1709 bis 1740, t. 2, Vienne : Hof- und Staats-Aerarial Druckerey.

Schöne, Andreas [2001], Die Leipziger ökonomische Sozietät von 17641825, dans Sächsische Aufklärung, édité par A. Klingenberg, Leipzig : Universitätsverlag, 73-91.

Sennewald, Rainer [2002], Die Stipendiatenausbildung von 1702 bis zur Gründung der Berrgakademie Freiberg 1765-1766, dans Technische Universität Bergakademie Freiberg, Festgabe zur 300. Jahrestag der Gründung der Stipendienkasse für die akademische Ausbildung im Berg- und Hüttenfach zu Freiberg in Sachsen, Freiberg : TU Bergakademie, 407-429. 
VoGEL, Jakob [2013], Aufklärung untertage : Wissenswelten des europäischen Bergbaus im ausgehenden 18. und frühen 19. Jahrhundert, dans Staat, Bergbau und Bergakademie : Montanexperten im 18. und frühen 19. Jahrhundert, édité par H. Schleiff \& P. Konecny, Stuttgart : Steiner, $13-31$.

Voigtel, Nikolaus [1686], Geometria Subterranea oder Markscheide-Kunst, Eisleben : Dietzel. 